\title{
Prominence modelling: from observed emission measures to temperature profiles
}

\author{
U. Anzer ${ }^{1}$ and P. Heinzel ${ }^{2,1}$ \\ 1 Max-Planck-Institut für Astrophysik, Karl-Schwarzschild-Str. 1, 85740 Garching, Germany \\ e-mail: ula@mpa-garching.mpg.de \\ 2 Astronomical Institute, Academy of Sciences of the Czech Republic, 25165 Ondřejov, Czech Republic \\ Received 11 October 2007 / Accepted 14 January 2008
}

\section{ABSTRACT}

\begin{abstract}
Aims. We outline the construction of prominence - corona transition region models based upon the observations of one particular prominence.

Methods. The differential emission measure curves from observations were approximated by simple analytical functions. On this basis we constructed the temperature curve and calculated the radiative losses, the gains by thermal conduction, and some estimates for the wave heating.

Results. The temperature curve was calculated in the range between $23000 \mathrm{~K}$ and $450000 \mathrm{~K}$. The resulting transition region can be divided into an inner region where the temperature is low and the radiative losses are very large, a part with a very steep temperature rise from $40000 \mathrm{~K}$ to around $250000 \mathrm{~K}$ over a width of only $500 \mathrm{~km}$, and an extended high temperature region. Both the conductive heating and our estimates for a possible wave cooling/heating peak very sharply in the region with the very large temperature gradient. The consequences for the energy balance are discussed.
\end{abstract}

Key words. Sun: prominences - Sun: transition region

\section{Introduction}

Solar prominences are cool structures within the very hot coronal environment. Typical prominence temperatures lie around $6000 \mathrm{~K}$ to $8000 \mathrm{~K}$, whereas the corona has temperatures between 1 and 2 million $\mathrm{K}$. This then implies that the temperature has to increase from prominence values to coronal ones in some region surrounding the prominence. This region is usually called the prominence-corona transition region (PCTR). The lines that are emitted in the temperature range of the PCTR lie in the UV and EUV part of the solar spectrum. Many of these lines have been observed in recent space missions. An early summary of some PCTR observations which used EUV lines was given by Orrall \& Schmahl (1976). They found that the intensities of these lines observed in the PCTR were systematically lower than those of the chromosphere-corona transition region. Later on Engvold et al. (1987) used UV line intensities and constructed an empirical model of the PCTR. Some more recent determinations of the PCTR on the basis of SOHO/SUMER and CDS observations were presented by Cirigliano et al. (2004).

Theoretical modelling of the PCTR was done by Chiuderi Drago et al. (1992), and by Anzer \& Heinzel (1999, 2000). These authors calculated the differential emission measure (DEM) as a function of the temperature and compared the results to the observationally obtained DEM curves. The modelling performed by the two groups was based on completely different configurations. Anzer \& Heinzel took 1D slab models with pressure equilibrium along the magnetic field, whereas Chiuderi Drago et al. studied an ensemble of horizontal magnetic flux tubes and calculated the superposition of all contributions.

A new set of very detailed measurements for one specific prominence has become available very recently; it is described in great detail in Parenti \& Vial (2007). In our present investigation we construct a detailed temperature profile for the entire PCTR. From this model one can calculate the local radiative losses in great detail. Since these losses have to be balanced by the corresponding heat input, our results give constraints on possible heating mechanisms operating in these regions. Our paper is organised in the following way: Sect. 2 presents the new observations, in Sect. 3 we give the calculated temperature profile, in Sect. 4 we derive the radiative losses in this region, Sect. 5 is devoted to the energy balance and Sect. 6 gives our conclusions.

\section{EUV observations of the prominence of 8 October 1999}

Based upon SOHO/SUMER observations Parenti et al. (2005) have produced a spectral atlas for one particular prominence in the wavelength range of $800-1250 \AA$. In this study the authors also included the spectral data which were obtained for the quiet Sun for comparison purposes. All the relevant measurements were taken on 8 October 1999. The set of observations was used by Parenti \& Vial (2007) to study in very great detail the PCTR of this particular prominence. The formation temperatures of the lines compiled in this atlas cover the range $4.0<\log T<6.3$. From the measured intensities of these lines one can calculate the differential emission measure of the entire PCTR and also of the neighbouring corona. The DEM is given by the relation

$\operatorname{DEM}(T)=n_{\mathrm{e}}^{2}\left(\frac{\mathrm{d} T}{\mathrm{~d} l}\right)^{-1}$,

where $l$ measures the total distance along the line of sight. The resulting $\operatorname{DEM}(T)$ curves obtained by Parenti \& Vial (2007) are 
sumarised in Fig. 2 of their paper. The top panel of this figure gives the DEM for the quiet Sun (QS), the two other panels are for two different parts of the same prominence (termed A_1 and A_2 by Parenti \& Vial). In the present investigation we are only interested in the prominence itself. One can also see from that figure that the scatter of the data points obtained for region A_2 is rather large. Therefore we concentrate in this paper on region A_1 and the DEM curve of the middle panel of Fig. 2. It should be noted that in this figure there are no data points below temperatures with $\log T=4.35$. Therefore the region between this temperature and the lower boundary of their plot at $\log T=4.2$ actually represents an extrapolation of the observations which may contain some uncertainties. For this reason we limit our investigation on the temperature region above $23000 \mathrm{~K}$. There is also the aspect that for the lower temperatures the assumption that all lines are optically thin may brake down.

Parenti \& Vial use an inversion method to generate the DEM curve on the basis of the observed line intensities. Their fitting curve is plotted together with individual data points in their Fig. 2. From these plots one can see that there is a very large scatter of the data points around the fitting curve. For this reason we use for our investigation a fit which is based on simple power laws. We take the following approximation:

$\operatorname{DEM}(T)=10^{23.4}\left(\frac{T}{23000}\right)^{-5.8}$

for the region of $23000 \mathrm{~K}<T<10^{5} \mathrm{~K}$ and

$\operatorname{DEM}(T)=10^{19.7} \frac{T}{10^{5}}$

for $10^{5} \mathrm{~K}<T<10^{6} \mathrm{~K}$.

This approximation lies within factors of 2 in the range of the curve given by Parenti \& Vial. Such small deviations are well within the uncertainties of the data. Therefore we take this simple form for our modelling. On this basis we then derive the temperature profile of the PCTR.

\section{Theoretical modelling of the prominence-corona transition region}

Our modelling of the PCTR is done for the simplest case of a $1 \mathrm{D}$ slab configuration. This kind of modelling is certainly an oversimplification. It is well known that prominences exhibit a wealth of fine structures (see e.g. Heinzel 2007). But in order to keep the modelling as simple as possible we adopt a 1D slab. In our Sect. 6 we shall briefly discuss some of the aspects which result from prominence fine structures and the associated PCTRs.

Such a simple slab model will have two PCTRs: one in the front of the prominence and one in the back. Both of them will contribute to the DEM derived from the observations. We do not know how the DEM is distributed over these two PCTR. Therefore we assume the simplest case in which the configuration is symmetric and both PCTRs are identical. Then each of them will contribute one half of the total DEM, and we get $\operatorname{DEM}_{1}(T)=1 / 2 \operatorname{DEM}(T)$, and this new $\operatorname{DEM}_{1}(T)$ then determines the structure of the two PCTRs.

To calculatve the temperature profile of the PCTR we now define the new function $F(T)$ given by

$F(T)=p_{\mathrm{e}}^{2}\left(\frac{\mathrm{d} T}{\mathrm{~d} l}\right)^{-1}$, where $p_{\mathrm{e}}$ represents the electron pressure. The function $F(T)$ is related to $\operatorname{DEM}(T)$ by the equation

$F(T)=\operatorname{DEM}(T)(k T)^{2}$.

Then the temperature gradient in each of the two PCTRs is obtained from the relation

$\frac{\mathrm{d} T}{\mathrm{~d} x}=\frac{2 p_{\mathrm{e}}^{2}}{F(T)}$

where we have introduced the coordinate $x$ which measures the distance away from the prominence. The origin for $x$ is taken at the inner boundary of our calculations where we have $T=23000 \mathrm{~K}$. Since in the entire PCTR $p_{\mathrm{e}}$ is approximately constant, Eq. (6) can be directly integrated to get the temperature profile in the PCTR. The assumption of constant pressure is only roughly valid. In particular in the innermost rather cool regions the pressure can increase towards the prominence. The consequences of this variation will be discussed later.

For region A_1 Parenti \& Vial give $n_{\mathrm{e}}=5.75 \times 10^{8} \mathrm{~cm}^{-3}$ at the position where one has $T=7 \times 10^{4} \mathrm{~K}$. This results in a value

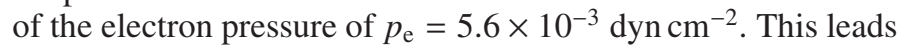
to the equation

$\frac{\mathrm{d} T}{\mathrm{~d} x}=\frac{2 p_{\mathrm{e}}^{2}}{\operatorname{DEM}(T) T^{2} k^{2}}$,

or

$\frac{\mathrm{d} T}{\mathrm{~d} x}=\frac{3.2 \times 10^{27}}{T^{2} \operatorname{DEM}(T)}$

Using Eqs. (2) and (8) we get for the inner parts of th PCTR the relation

$\frac{\mathrm{d} T}{\mathrm{~d} x}=6.41 \times 10^{-22} T^{3.8}$.

The integration then produces the temperature profile of this region as

$$
T=\frac{1}{\left(23000^{-2.8}-1.79 \times 10^{-21} x\right)^{1 / 2.8}},
$$

where $x=0$ represents the inner boundary of the PCTR placed at $T=23000 \mathrm{~K}$. One also finds that the temperature of $10^{5} \mathrm{~K}$ is reached at a distance of $x_{1}=3370 \mathrm{~km}$.

By the same procedure we obtain for the outer part

$\frac{\mathrm{d} T}{\mathrm{~d} x}=\frac{6.4 \times 10^{12}}{T^{3}}$

This results in

$T=\left[10^{20}+2.64 \times 10^{13}\left(x-x_{1}\right)\right]^{1 / 4}$.

If we consider all radiation that is emitted at temperatures above around $4.5 \times 10^{5} \mathrm{~K}$ as being of coronal origin, then the PCTR extends only to this temperature, and we can calculate its spatial extension which amounts to $x_{2}=15500 \mathrm{~km}+3370 \mathrm{~km}=$ $18870 \mathrm{~km}$. The temperature profile of this entire region is plotted in Fig. 1. 


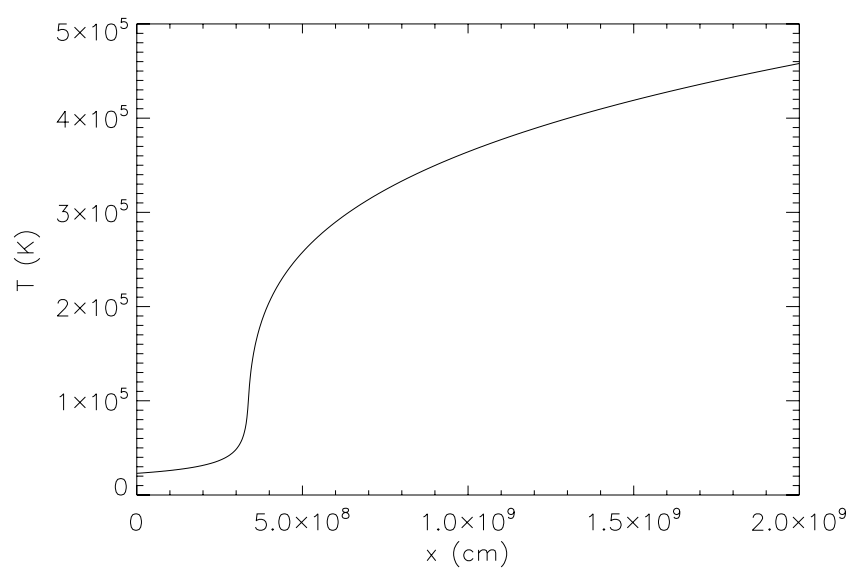

Fig. 1. Plot of the temperature variation along the line of sight.

\section{Radiative losses in the transition region}

On the basis of the temperature variation in the PCTR calculated in the previous section we can now determine the local radiative losses that are present in this region. Since we consider only those parts of the PCTR where the temperature is above $23000 \mathrm{~K}$ we can use the formula for optically thin losses given by

$L(T)=n_{\mathrm{e}}^{2} \Lambda(T)$.

With the relation $n_{\mathrm{e}}=p_{\mathrm{e}} /(k T)$ we get

$L(T)=\left(\frac{p_{\mathrm{e}}}{k}\right)^{2} \frac{\Lambda(T)}{T^{2}}$.

The loss function $\Lambda(T)$ has been estimated by many different authors (e.g. Hildner 1974). But with the new set of atomic data collected in the CHIANTI programme one can now calculate more detailed and accurate loss rates. Figure 2 shows the loss function for two cases: one for photospheric abundances (solid curve), and one for coronal values (dashed curve). Both of them were kindly provided to us by Ken Dere. Since we still do not understand the process of the prominence formation in sufficient detail we cannot say which set of abundances is the correct one. If prominences are formed by injection or by the syphon mechanism then photospheric abundances should be taken; but if the prominences result from condensations out of the corona then the latter should apply. Here we take photospheric abundances which were also used in Parenti \& Vial for the determination of the $\operatorname{DEM}(T)$. The photospheric abundances which we used here were given by Grevesse \& Sauval (1998). But as one can see from our Fig. 2 it is not very crucial which abundances one takes to calculate the losses. In general the differences are less than a factor 1.5; only at temperatures which lie above $450000 \mathrm{~K}$ these differences will become substantial. In our study we ignore those parts of the DEM curve which are above $450000 \mathrm{~K}$ because these parts probably belong to the ambient corona. For this reason the loss curve with photospheric abundances seems quite adequate. For the analytical investigation of the PCTR losses we use an approximate loss curve which is given by piece-wise power laws of the form

$\Lambda(T)=a_{n}\left(\frac{T}{T_{n}}\right)^{v_{n}}$.

The values for $T_{n}, a_{n}$ and $v_{n}$ for the range of temperatures used here are summarised in Table 1.

Inserting now the observed value of $p_{\mathrm{e}}=5.6 \times 10^{-3} \mathrm{dyn} \mathrm{cm}^{-2}$ into Eq. (14) and using Eq. (15) with the parameters specified

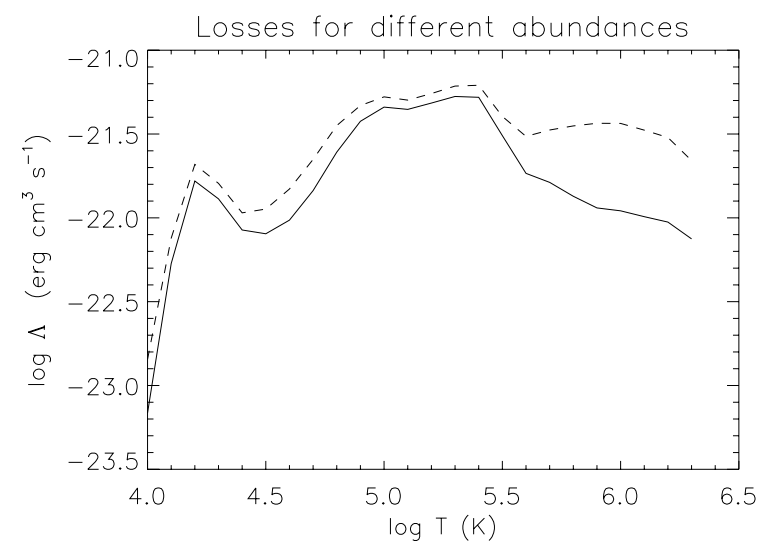

Fig. 2. Loss function $\Lambda(T)$ for photospheric abundances (solid line) and coronal abundances (dashed line).

Table 1. Fitting parameters for loss funtion.

\begin{tabular}{rrrr}
\hline \hline Temperature range & $T_{n}$ & $a_{n}$ & $v_{n}$ \\
\hline $23000-35000$ & 23000 & $10^{-21.96}$ & -1.03 \\
$35000-79000$ & 35000 & $10^{-22.15}$ & +2.12 \\
$79000-250000$ & 79000 & $10^{-21.40}$ & +0.20 \\
$250000-450000$ & 250000 & $10^{-21.30}$ & -1.80 \\
\hline
\end{tabular}

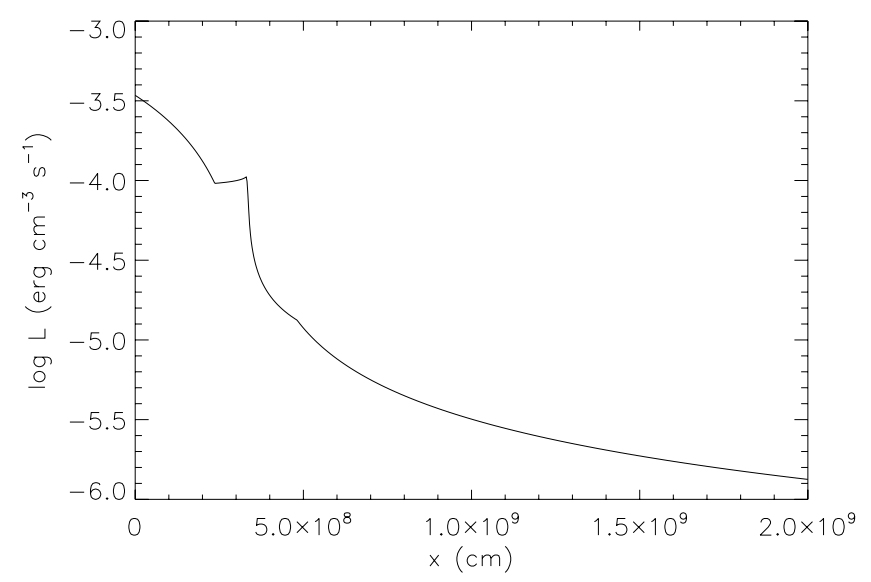

Fig. 3. Local radiative losses for the part of the PCTR with $T<$ $450000 \mathrm{~K}$.

in Table 1 we obtain the radiative losses as a function of the local temperature. With the help of Eqs. (10) and (12) we then transform these losses to the spatial variable $x$. This loss curve is shown in Fig. 3.

The curve shows that the losses per unit volume are two orders of magnitude larger in the innermost parts of the PCTR as compared to the outer, high temperature, regions. The maximum losses amount to around $3 \times 10^{-4} \mathrm{erg} \mathrm{cm}^{-3} \mathrm{~s}^{-1}$, and drop sharply to values of $10^{-5} \mathrm{erg} \mathrm{cm}^{-3} \mathrm{~s}^{-1}$ over a distance of only $5000 \mathrm{~km}$. This result has important consequences for the energy balance inside the PCTR. The loss curve also shows a small secondary peak around $3300 \mathrm{~km}$. This peak is due to the increase in $L(T)$ in the range between $35000 \mathrm{~K}$ and $79000 \mathrm{~K}$. Because of the extremely large temperature gradient in this part of the PCTR the peak is squeezed into a very narrow region in $x$. This will require a concentrated local heating of this part of the PCTR. The consequences of these aspects for any heating model will be discussed in the next section. 


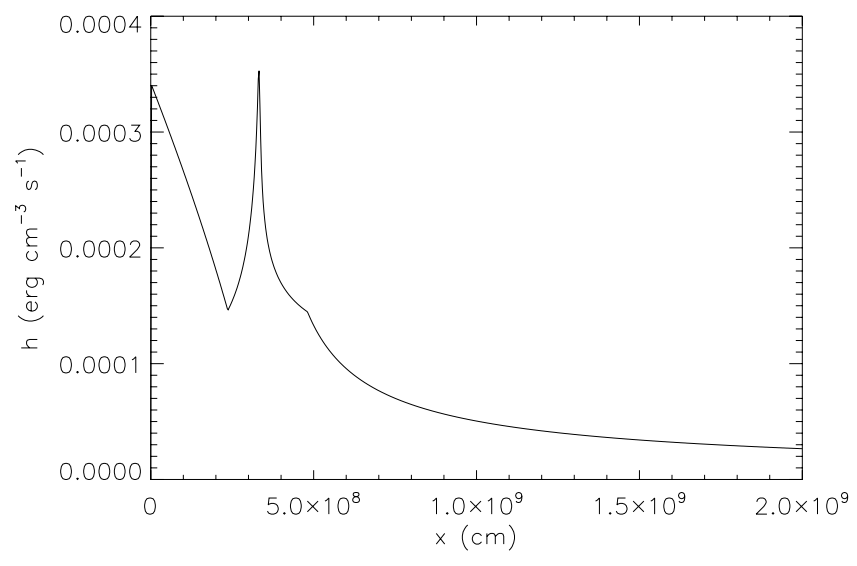

Fig. 4. The heating function $h(x)$ as obtained for the inner parts of the PCTR.

\section{Requirements for energy balance}

In order to obtain a PCTR which is in a steady state the local radiative losses have to be balanced by some (still unspecified) heating mechanism. This heating function $H(x)$ therefore must be given by the relation

$H(x)=L[T(x)]$,

where $L$ is determined by Eqs. (14) and (15). In the following we assume that the heating is proportional to the density, $\rho$, and for a fully ionised plasma also to $n_{\mathrm{e}}$. With this we get

$H(x)=\frac{n_{\mathrm{e}}}{n_{\mathrm{e} 0}} h(x)$,

or

$H(x)=\frac{p_{\mathrm{e}}}{p_{\mathrm{e} 0}} \frac{T_{0}}{T} h(x)$.

We take again for the PCTR a constant electron pressure, and with this we arrive at the following relation

$h(x)=\frac{T}{T_{0}} L[T(x)]$.

With $T_{0}=23000 \mathrm{~K}$ we can now determine the heating function $h(x)$. The curve for $h(x)$ is shown in Fig. 4.

This heating function decreases linearly in the inner region with $h=3.5 \times 10^{-4}(4100-x)$. Around the position $x=3300 \mathrm{~km}$ there is a secondary, rather narrow, peak of $h$. This peak directly corresponds to the one in the loss curve of Fig. 3, but because of the additional factor of $T$ in Eq. (19) the peak is much stronger in this plot. This secondary peak could be related to the heating by thermal conduction.

\subsection{Heating by thermal conduction}

One possible contribution to the heating and cooling of the PCTR is the divergence of the conductive heat flow, $F_{\mathrm{c}}$. This heat flow occurs primaryly in the direction of the magnetic field. It is given by the relation

$F_{\mathrm{c}}=\kappa_{1} T^{5 / 2} \frac{\mathrm{d} T}{\mathrm{~d} s}$

where $\kappa_{1}=10^{-6}$ (in cgs units) and $s$ measures the distance along the field. It is related to $x$ by $s=x / \cos \alpha$, where $\alpha$ is the angle between the line of sight and the field direction. The value of

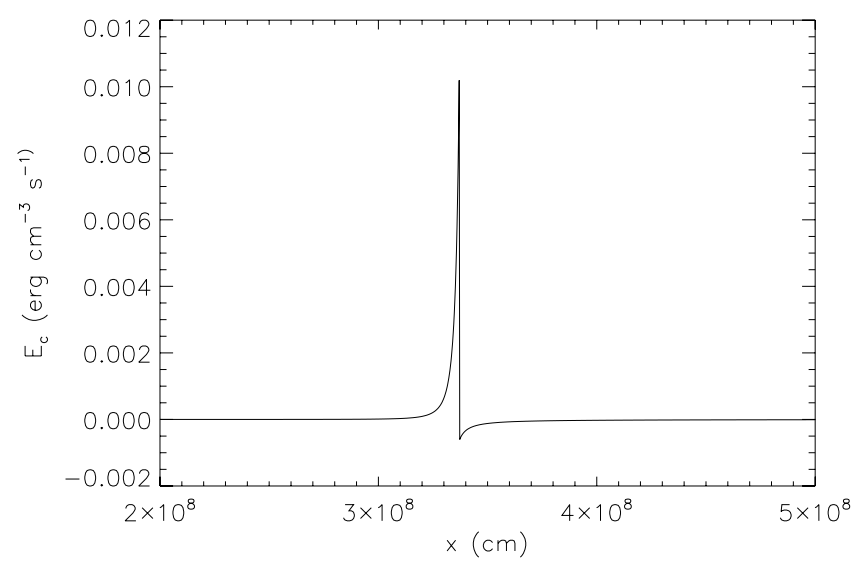

Fig. 5. Conductive gains and losses for the case of $\alpha=0$.

$\alpha$ depends both on the magnetic shear and on the prominence orientation. Since the energy deposition by thermal conduction is given by $\mathrm{d} F_{\mathrm{c}} / \mathrm{d} s$ we obtain for the conductive heating (respectively cooling) the equation

$E_{\mathrm{c}}=(\cos \alpha)^{2} 10^{-6} \frac{\mathrm{d}}{\mathrm{d} x}\left(T^{5 / 2} \frac{\mathrm{d} T}{\mathrm{~d} x}\right)$.

Making now use of the Eqs. (9) and (11) we arrive at the final equations for the heating and cooling.

$E_{\mathrm{c}}=(\cos \alpha)^{2} 2.59 \times 10^{-48} T^{9.1}$

for $T<100000 \mathrm{~K}$ and

$E_{\mathrm{c}}=-(\cos \alpha)^{2} 2.05 \times 10^{19} T^{-4.5}$

for $T>10^{5} \mathrm{~K}$. The quantity $E_{\mathrm{c}}$ is plotted in Fig. 5 for $\alpha=0$. Note: in this figure and the following we plot (different from the previous set of figures) only those parts of the PCTR where the effects of heating/cooling become sufficiently large.

The maximum conductive heating occurs where the gradient $\mathrm{d} T / \mathrm{d} x$ is largest; this is where the function $T^{2} \operatorname{DEM}(T)$ has its minimum, i.e. around $T=10^{5} \mathrm{~K}$. The secondary (small) peak in the radiative loss curve of Fig. 3 is located at the maximum of the function $\Lambda(T) / T^{2}$, which for our loss function is around $T=80000 \mathrm{~K}$. Therefore the peak is approximately at the position of maximum conductive heating. One also sees that the maximum heating amounts to $10^{-2} \mathrm{erg} \mathrm{cm}^{-3} \mathrm{~s}^{-1}$, and the secondary peak of radiative losses is only $10^{-4} \mathrm{erg} \mathrm{cm}^{-3} \mathrm{~s}^{-1}$ (see Fig. 3). This would imply that there is too much heating in this part of the PCTR. But this region is extremely narrow and one finds that the integrated heating in this region is actually comparable to the total losses between temperatures of $35000 \mathrm{~K}$ and $80000 \mathrm{~K}$. This implies that if this heating could be distributed over a somewhat larger region it could produce the required energy balance. But there is also the point that the heating scales as $(\cos \alpha)^{2}$ and therefore any inclination angles above $75^{\circ}$ would reduce the heating efficiency by more than $1 / 15$. This will lead to large uncertainties in the energetics of this part. But on the other hand it is evident that conductive heating is completely insufficient to balance the losses in the cooler parts.

\subsection{Wave heating}

An additional heating (or cooling) mechanism could be the energy deposition (or extraction) by travelling waves. We consider only Alfvén waves, because sound waves would be much less 


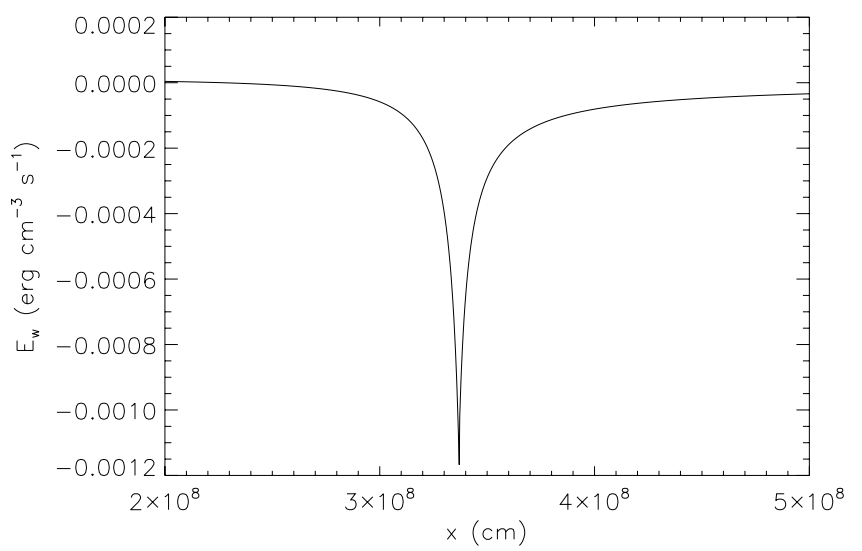

Fig. 6. Heating and cooling by Alfvén waves.

efficient. Parenti \& Vial (2007) determined the observed nonthermal velocities that are present in the PCTR. Their results are given in Fig. 1 of that paper. We again concentrate on region A_1 of their investigation. The velocities, $\xi$, plotted in this figure show a very large scatter. But for our analytical treatment we assume a simple fitting to the data points in the temperature range between $23000 \mathrm{~K}$ and $4.5 \times 10^{5} \mathrm{~K}$ given by

$\xi=2.5 \times 10^{6}(0.36 \log T-1)$,

where $\xi$ is given in $\mathrm{cm} \mathrm{s}^{-1}$ and $T$ in degree Kelvin. The amount of heating is determined by the divergence of the wave flux. For our 1D PCTR we then obtain

$E_{\mathrm{w}}=-\frac{\mathrm{d}}{\mathrm{d} x}\left(\frac{1}{2} \rho \xi^{2} v_{x}\right)$.

In our notation $v_{x}>0$ represents outgoing waves and $v_{x}<0$ incoming waves. The Alfvén velocity can be calculated from

$v_{x}=\frac{B}{\sqrt{(4 \pi \rho)}}$.

For a fully ionised hydrogen plasma $\rho$ is related to the electron pressure by

$\rho=\frac{2 \bar{m} p_{\mathrm{e}}}{k T}$,

where $\bar{m}$ is the mean molecular mass $\left(0.8 \times 10^{-24} \mathrm{~g}\right.$ in our case $)$. For $\rho=8 \times 10^{-11} T^{-1}$ and assuming a magnetic field of $10 \mathrm{G}$ (smaller fields will give correspondingly lower rates) we determine the heating/cooling rate for inward travelling waves as

$E_{\mathrm{w}}=-5.09 \times 10^{6}(\log T-4.53)(\log T-2.78) T^{-3 / 2} \frac{\mathrm{d} T}{\mathrm{~d} x}$.

The gradient $\mathrm{d} T / \mathrm{d} x$ for the entire PCTR is given in our Eqs. (9) and (11). This leads to the final equations for the heating/cooling rates

$E_{\mathrm{w}}=-3.26 \times 10^{-15}(\log T-4.53)(\log T-2.78) T^{2.3}$

and

$E_{\mathrm{w}}=-3.26 \times 10^{19}(\log T-4.53)(\log T-2.78) T^{-4.5}$.

The resulting heating/cooling function is shown in Fig. 6.

This figure shows that over a large fraction of the PCTR the dominant effect of these waves is a strong cooling. Only in the innermost part one has some heating. Therefore these waves would increase the problem with the energy balance rather than solving it. The reason for this behaviour is the fact that the assumed variation of $\xi$ with $T$ is not large enough to compensate for the $T^{-1 / 2}$ decrease of the quantity $\rho v_{x}$. Only for such $\xi$ which increase faster than $T^{1 / 4}$ one would obtain some heating of the PCTR. Because of the large scatter in the data points of Parenti \& Vial (2007) this is not completely excluded, but there is also no strong evidence for such a behaviour.

Appart from the uncertainty of the sign of the energy contribution there are also some other problems:

i. it is not obvious that the observed non-thermal motions are really the amplitudes of some wave motions. They could also be caused by random motions related to turbulence;

ii. in the case of travelling waves one would need a very efficient damping mechanism in order to convert the energy. This is completely open so far.

Therefore it is unclear to what extent travelling waves could contributee to the solution of the energy problem in PCTRs.

\section{Conclusions}

The very detailed measurements of the prominence observed on 8 October 1999 allow a reliable construction of the PCTR for this particular prominence. But we expect other prominences to be similar, and therefore these results should be quite general. Using 1D slab models and assuming a constant electron pressure in the entire PCTR one can derive a detailed temperature profile over the range of $23000 \mathrm{~K}$ to $10^{6} \mathrm{~K}$. The regions above, say $450000 \mathrm{~K}$, probably belong to the surrounding corona, and therefore are not considered in our analysis. The most striking result from the present modelling is the presence of a very extended region of low temperatures between $23000 \mathrm{~K}$ and $50000 \mathrm{~K}$ (see Fig. 1). The width of this region can be estimated to be around $3500 \mathrm{~km}$. One also finds that the radiative losses in this innermost part dominate by orders of magnitude over those calculated for the remainder of the PCTR.

These losses have to be balanced by some form of heating. We have assumed that the heating is in the form of mechanical heating which scales as the density, $\rho$. In this case we found that the specific heating has to increase inwards by a factor 2.5 over a distance of $2500 \mathrm{~km}$. But so far no reasonable heating mechanism which fulfills this requirement has been found. We have also estimated other forms of heating, e.g. by thermal conduction, or by the dissipation of Alfvén waves. We found that both these mechanisms are inadequate to heat the inner parts of the PCTR, because in these regions the heating rates are several orders of magnitude too low. But heating by thermal conduction could provide enough energy to balance the losses of the small secondary peak around $3300 \mathrm{~km}$ visible in Fig. 3. But there the thermal heating will have to be smeard out over a somewhat larger region. The effects of heating (or more likely cooling) by Alfvén waves are still unclear since the present observations do not allow a sufficiently accurate determination of the variation of the non-thermal velocities with temperature in the PCTR. Moreover it is doubtful that the observed velocities really represent the amplitudes of travelling waves. Therefore one has to conclude that at present the problem of the PCTR heating is still unsolved.

Our model is idealised in several ways:

I. We have assumed a constant electron pressure for the entire PCTR. This is certainly correct in the outer parts of the 
PCTR where both the ionisation degree of hydrogen is constant (close to unity) and the pressure scale heights are very large. In the inner, cooler parts the ionisation degree will decrease somewhat, but this effect is rather small. Much more important is the fact that in these regions the pressure scale heights become small. If now the magnetic field has some non-negligible inclination with the horizontal plane then the pressure will increase inwards. In this case our Eq. (6) cannot be integrated analytically. But we did not follow up on this problem; instead we made some rough estimates of the effect. On this basis we found that if the inclination angle is around $10^{\circ}$ to $20^{\circ}$ then the width of the innermost part will be reduced by a factor of the order 2 with respect to our analytical result. In the case that the magnetic dips are even shallower our earlier results remain approximately correct.

II. The other point is that our models are purely onedimensional, describing the PCTR as vertical slabs. But one knows that prominences exhibit a wealth of fine structures (for a discussion, see e.g. Heinzel 2007). Each of these prominence elements will have its own PCTR. Then the observed DEM will be the sum of all the PCTRs within tha area of resolution. Under these circumstances one can no longer obtain a simple geometric description of thes PCTRs. The interpretation then will become strongly model-dependent. Some rather crude fine structure modelling of a particular geometry has been presented by Chiuderi Drago et al. (1992), and also by Cirigliano et al. (2004). These authors assumed an ensemble of horizontal magnetic flux tubes filled with cool plasma, and each of these flux tubes has its own prescribed PCTR. Another type of fine structure models consisting of vertical threads has recently been developed by Gunár et al. (2007). But that paper is devoted to the Lyman lines of hydrogen which are formed in cooler regions, and therefore is of no direct consequence for our present modelling. We plan to extend this type of modelling of fine structures to the higher temperatures of the outer regions of the PCTRs and then compare the theoretical DEM curves with the ones obtained from the observations. Such an approach could give us more constraints for our vertical thread modelling. This will be done in a future paper.

Acknowledgements. We thank Ken Dere for his help with the CHIANTI data set. Part of this work was supported by the ESA-PECS project No. 98030. We also thank the referee, S. Parenti, for her valuable comments.

\section{References}

Anzer, U., \& Heinzel, P. 1999, A\&A 349, 974

Anzer, U., \& Heinzel, P. 2000, A\&A 358, L75

Chiuderi Drago, F., Engvold, O., \& Jensen, E. 1992, Sol. Phys., 139, 47

Cirigliano, D., Vial, J.-C., \& Rovira, M. 2004, Sol. Phys., 223, 95

Engvold, O., Kjeldseth-Moe, O., Bartoe, J.-D. F., \& Brueckner, G .E. 1987, in Small-Scale Plasma Processes, ed. B. Battrick, \& E. J. Rolfe, ESA SP-275, 21

Grevesse, N., \& Sauval, A. J. 1998, Space Sci. Rev., 85, 161

Gunár, S., Heinzel, P., Schmieder, B., Schwartz, P., \& Anzer, U. 2007, A\&A, 472,929

Heinzel, P. 2007, in The Physics of Chromospheric Plasmas, ed. P. Heinzel, I. Dorotovič, \& R. J. Rutten, ASP, 368, 271

Hildner, E. 1974, SP, 35, 123

Orrall, F. Q., \& Schmahl, E. J. 1976, SP, 50, 365

Parenti, S., \& Vial, J.-C. 2007, A\&A, 469, 1109

Parenti, S., Vial, J.-C., \& Lemaire, P. 2005, A\&A, 443, 679 